\title{
COUNTING SEMIREGULAR PERMUTATIONS WHICH ARE PRODUCTS OF A FULL CYCLE AND AN INVOLUTION
}

\author{
D. M. JACKSON
}

\begin{abstract}
Character theoretic methods and the group algebra of the symmetric group are used to derive properties of the number of permutations, with only $p$-cycles, for an arbitrary but fixed $p$, which are expressible as the product of a full cycle and a fixed point free involution. This problem has application to single face embeddings of $p$-regular graphs on surfaces of given genus.
\end{abstract}

1. Introduction. A permutation is said to be $p$-semiregular if each cycle in its disjoint cycle decomposition has length $p$. The identity, a fixed point free involution and a full (i.e. of length $N$ ) cycle on $N$ symbols are instances of 1-semiregular, 2semiregular and $N$-semiregular permutations, respectively. For permutations on $N=k p$ symbols, let $e(k, p)$ be the number of $p$-semiregular permutations which are expressible as the product of an arbitrary but fixed full cycle and a fixed point free involution. In this paper we investigate the properties of this number. The problem has application to the study of 2-cell embeddings of graphs and hypergraphs on an orientable 2-manifold (Machi [5]). It was prompted by the work of Bayer and Morrison [6] on singularities in surfaces, where they considered permutations with a prescribed number of cycles, expressible in this form. This and a generalization were considered in [4]. The present question is a restriction of Bayer and Morrison's problem to the $p$-semiregular case.

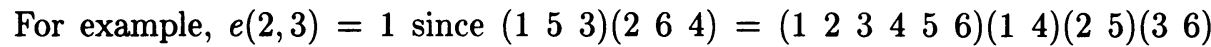
is the unique 3-semiregular permutation in $\mathbf{S}_{6}$ which is the product (carried out from right to left) of the fixed cycle ( $\left.\begin{array}{llllll}1 & 2 & 3 & 4 & 5 & 6\end{array}\right)$ and a fixed point free involution. It is a feasible but laborious hand calculation to show that $e(3,4)=45$. One of these 45 permutations is $\left(\begin{array}{llll}1 & 8 & 11 & 6\end{array}\right)\left(\begin{array}{llll}2 & 5 & 12 & 7\end{array}\right)\left(\begin{array}{llll}3 & 10 & 9 & 4\end{array}\right)$ since this is equal to $(12 \cdots 12)(1 \quad 7)(24)(3 \quad 9)(511)(612)(8$ 10). These hand calculations agree with Corollaries 5.2 and 5.1 respectively. Although embeddings of maps are peripheral to the problem, the maps corresponding to these two cases are given in the Appendix, together with diagrams giving the faces explicitly.

We use, without further explanation, combinatorial and algebraic facts about the group algebra $\mathbf{C S}_{N}$ of the symmetric group $\mathbf{S}_{N}$ on $N$ symbols over the complex field C. These facts are to be found in [4], together with notational conventions and the necessary information about the characters of $\mathbf{S}_{N}$. Although $\mathbf{C S} \mathbf{S}_{N}$ is particularly suited, in combinatorial theory, to the study of the cycle structure of products of permutations, any nontrivial use of it leads to the introduction of characters. Restricted sums of these are difficult to deal with in general, and it is often a

Received by the editors October 24, 1986.

1980 Mathematics Subject Classification (1985 Revision). Primary 05A15, 20C15; Secondary 57 N37. 
delicate matter to use them to elicit further combinatorial information. Instances in combinatorial theory in which character theoretic arguments have been used include factorization of permutations into full cycles (Stanley [10]), random permutations generated by random transpositions (Diaconis and Shahshahani [3]), asymptotic estimation of the number of homomorphisms of a particular type from a finitely generated free monoid to a finite group (Thompson [11]) and association schemes (Bannai and Ito [1]). See also Rothaus and Thompson [8].

First we express $e(k, p)$ as a multiple of a particular character sum, which is denoted by $T(k, p)$. We then demonstrate that $T(k, p)$ is zero except for the two cases $T(2 n+1,4 q)$ and $T(4 n+2,2 q+1)$, where $n, q$ are nonnegative integers here and throughout. Explicit expressions are obtained for these two cases by considering an integral representation of $T(k, p)$. This is carried out in $\S \S 3$ and 4 , respectively, for these two cases. The integral representations are further used in $\S 5$ to derive expressions for $T(4 n+2,3)$ and $T(2 n+1,4)$, and again in $\S 6$ to show that $T(2 n+1,8)$ and $T(4 n+2,5)$ satisfy 3 -term linear recurrence equations. This is achieved by expressing each in terms of the hypergeometric series ${ }_{2} F_{1}$ (namely, Gauss' function), and by using the contiguous relations for this function.

It would be desirable to have a direct (combinatorial) proof of the recurrence equations for $T(2 n+1,8)$ and $T(4 n+2,5)$, which appears to be possible because they have only three terms. However, among the coefficients of these terms are polynomials of degrees four and seven with large coefficients. This may mean that such a proof is very difficult.

It is convenient to have the following notation readily to hand. If $\theta$ is a partition of $N$ we write $\theta \vdash N$. The set of all partitions of $N$ is a natural index set for the conjugacy classes of $\mathbf{S}_{N}$, in which each element of the conjugacy class $\mathbf{C}_{\theta}$ corresponding to $\theta=\left[1^{i_{1}} 2^{i_{2}} \ldots\right]$ has $i_{j} j$-cycles for $j=1, \ldots, N$. The size of $\mathbf{C}_{\theta}$ is denoted by $h^{\theta}$ and is equal to $N ! /\left(1^{i_{1}} i_{1} ! 2^{i_{2}} i_{2} ! \cdots\right)$. In particular, $h^{[k p]}=(N-1)$ !, $h^{\left[p^{k}\right]}=N ! /\left(p^{k} \cdot k !\right)$ and $h^{\left[2^{k p / 2}\right]}=N ! /\left(2^{k p / 2} \cdot(k p / 2) !\right)$. The irreducible (ordinary) character associated with $\mathbf{C}_{\theta}$ is denoted by $\chi^{\theta}$, its value at any element of $\mathbf{C}_{\alpha}$, where $\alpha \vdash N$, is denoted by $\chi_{\alpha}^{\theta}$, and its degree is denoted by $f^{\theta}\left(=\chi_{\left[1^{N}\right]}^{\theta}\right)$.

All subsequent computations take place in $\mathbb{C}[[x]]$, the ring of formal power series in the indeterminate $x$ with coefficient ring $\mathbb{C}$. The coefficient operator on this ring is defined by

$$
\left[x^{n}\right]: \mathbb{C}[[x]] \rightarrow \mathbb{C}: \sum_{i \geq 0} f_{i} x^{i} \mapsto f_{n} .
$$

Integration on this ring is to be taken formally. As usual, $\mathbf{R} c$ denotes the real part of $c \in \mathbb{C}$.

For a nonnegative integer $m$, the Pochhammer symbol $(a)_{m}$ is defined by $(a)_{m}=$ $a(a+1)(a+2) \cdots(a+m-1)$. In simplifying hypergeometric sums over a variable $j$, say, we use the (now) familiar device of reducing each instance of the Pochhammer symbol to the canonical form in which $a$ is independent of $j$, and $m$ contains only multiples of $j$. This may be achieved by using the elementary transformations, among which are included: $(a)_{d+j}=(a)_{d}(a+d)_{j} ;(a)_{2 j}=\left(\frac{1}{2} a\right)_{j}\left(\frac{1}{2}+\frac{1}{2} a\right)_{j} 4^{j}$; $(a)_{d-j}=(-1)^{j}(a)_{d} /(1-a-d)_{j}$ where $d, j$ are nonnegative integers. In the interests of brevity, details of the simplification of hypergeometric expressions are condensed to a few salient steps. 
The following constants occur frequently:

$$
K(k, p)=\frac{1}{2} k p \text { and } \Delta(k, p)=4^{K}(2 K) ! / K !^{2},
$$

which are abbreviated in the generic case to $K$ and $\Delta$, respectively. Throughout, $n, k$ and $p$ are nonnegative integers and $2 \mid k p$, so $K$ is a nonnegative integer.

2. The character sum and its two cases. Throughout, we shall be concerned with the following character sum.

DEFINITION 2.1 .

$$
T(k, p)=\sum_{\theta \vdash k p} \frac{1}{f^{\theta}} \chi_{[k p]}^{\theta} \chi_{\left[p^{k}\right]}^{\theta} \chi_{\left[2^{k p / 2}\right]}^{\theta}
$$

The combinatorial number $e(k, p)$, which is the subject of this paper, can be expressed in terms of this character sum as follows.

PROPOSITION 2.2.

$$
e(k, p)=h^{\left[2^{k p / 2}\right]} h^{\left[p^{k}\right]} T(k, p) /(k p) !
$$

PROOF. Special case of $[4$, Corollary 2.5].

Another combinatorial number may be expressed in terms of $T(k, p)$. Let $c(k, p)$ be the number of ways of expressing a $p$-semiregular permutation as the product of a full cycle and a fixed point free involution. Then, from the proof of Lemma 2.3 of $[\mathbf{4}]$,

$$
c(k, p)=h^{\left[2^{k p / 2}\right]} h^{[k p]} T(k, p) /(k p) ! .
$$

Proposition 4.1 and Corollaries 3.8 and 3.9 of [4] may be used to obtain an expression for the character sum solely in terms of operations on univariate formal power series.

LEMMA 2.3.

$$
\begin{aligned}
& T(k, p)=\sum_{i=0}^{k p-1}(-1)^{i}\left(\begin{array}{c}
k p-1 \\
i
\end{array}\right)^{-1}\left\{\left[x^{i}\right](1+x)^{-1}\left(1-x^{2}\right)^{k p / 2}\right\} \\
& \cdot\left[x^{i}\right](i+x)^{-1}\left\{1-(-x)^{p}\right\}^{k} .
\end{aligned}
$$

We first use this lemma to establish when the character sum is zero.

PROPOSITION 2.4. Unless $p \equiv 0 \bmod 4$ and $k \equiv 1 \bmod 2$, or $p \equiv 1 \bmod 2$ and $k \equiv 2 \bmod 4$, then $T(k, p)=0$.

PrOOF. Suppressing the dependence on $k$ and $p$, we let $s_{i}=\left[x^{i}\right] f$ where $f=(1+x)^{-1}\left(1-x^{2}\right)^{k p / 2}$ and $t_{i}=\left[x^{i}\right] g$ where $g=(1+x)^{-1}\left\{1-(-x)^{p}\right\}^{k}$. Clearly, $f, g \in \mathbb{C}[x]$ and are polynomials of degree $k p-1$ in $x$. Moreover, $f(x)=$ $(-1)^{k p / 2} x^{k p-1} f\left(x^{-1}\right)$ so $s_{k p-i-1}=(-1)^{k p / 2} s_{i}$, and $g(x)=(-1)^{k(p+1)} x^{k p-1} g\left(x^{-1}\right)$ so $t_{k p-i-1}=(-1)^{k(p+1)} t_{i}$. We conclude, from Lemma 2.3 , that

$$
T(k, p)=(-1)^{k-1+k p / 2} T(k, p),
$$

whence

$$
T(k, p)=\frac{1}{2}\left\{1-(-1)^{k+k p / 2}\right\} T(k, p) .
$$


But $1-(-1)^{k+k p / 2}=0$ if (a) $k \equiv 0 \bmod 4 ;$ or if (b) $k \equiv 0 \bmod 2$ and $p \equiv 0$ $\bmod 2$; or if $(\mathrm{c}) k \equiv 1 \bmod 2$ and $p \equiv 2 \bmod 4$. The result follows by recalling that $2 \mid k p$.

We conclude from this proposition that there are two possibilities, namely, $T(2 n+1,4 q)$ and $T(4 n+2,2 q+1)$, for $T(k, p)$, and that the remaining cases are zero. These cases are considered separately in the next two sections.

Expressions for the combinatorial numbers $e(k, p)$ and $c(k, p)$ are readily obtained from Proposition 2.2, the remark which follows it, and the expression for $h^{\theta}$ given in $\S 1$.

3. An integral representation for the character sum $T(2 n+1,4 q)$. To obtain further information about $T(k, p)$ we derive an integral representation for it. In doing so, extensive use is made of the next proposition concerning the beta integral.

PrOPOSITION 3.1. Let $\Phi_{n+p}(p)=((2 n) ! / n !)((2 p) ! / p !)$. Then

$$
\Phi_{n+p}(p)=\frac{2}{\pi} 4^{n+p}(n+p) ! \int_{0}^{\pi / 2} \sin ^{2 n} x \cos ^{2 p} x d x .
$$

The next theorem gives an integral representation for this case.

THEOREM 3.2 .

$$
T(k, 4 q)=\frac{2}{\pi} \Delta \int_{0}^{\pi / 2}\left(\cos ^{4 q} x-\sin ^{4 q} x\right)^{k} \cos 2 x d x .
$$

PROOF. From Lemma 2.3

$$
\begin{aligned}
T(k, 4 q)=\sum_{i=0}^{4 k q-1}(-1)^{i}\left(\begin{array}{c}
4 k q-1 \\
i
\end{array}\right)^{-1}\left\{\left[x^{i}\right](1+x)^{-1}\left(1-x^{2}\right)^{2 k q}\right\} & \cdot\left[x^{i}\right](1+x)^{-1}\left(1-x^{4 q}\right)^{k} .
\end{aligned}
$$

Let $\mathbf{N}_{i}=\{0,1,2, \ldots, i\}$. Then the mapping

$$
\rho: \mathbf{N}_{k-1} \times \mathbf{N}_{2 q-1} \times \mathbf{N}_{1} \rightarrow \mathbf{N}_{4 q k-1}:(j, l, m) \mapsto 4 q j+2 l+m
$$

is bijective. Moreover, if $\rho^{-1}(i)=(j, l, m)$ then

$$
\left[x^{i}\right](1+x)^{-1}\left(1-x^{2}\right)^{2 k q}=(-1)^{l+m}\left(\begin{array}{c}
2 k q-1 \\
2 j q+l
\end{array}\right)
$$

and

$$
\left[x^{i}\right](1+x)^{-1}\left(1-x^{4 q}\right)^{k}=(-1)^{j+m}\left(\begin{array}{c}
k-1 \\
j
\end{array}\right)
$$

so

$$
\begin{aligned}
T(k, 4 q) & =\frac{K !}{(2 K) !} \sum_{j=0}^{k-1}(-1)^{j}\left(\begin{array}{c}
k-1 \\
j
\end{array}\right) \sum_{l=0}^{2 q-1}(-1)^{l} \sum_{m=0}^{1}(-1)^{m} \Phi_{2 k q}(2 q j+l+m) \\
& =\frac{K !}{(2 K) !} \sum_{j=0}^{k-1}(-1)^{j}\left(\begin{array}{c}
k-1 \\
j
\end{array}\right) \sum_{l=0}^{2 q} \alpha_{l} \Phi_{2 q k}(2 q j+l)
\end{aligned}
$$


where $\alpha_{l}=1$ if $l=0$ or $2 q$ and $\alpha_{l}=2(-1)^{l}$ if $0<l<2 q$. From Proposition 3.1

$$
\begin{aligned}
T(k, 4 q)=\frac{2}{\pi} \Delta \int_{0}^{\pi / 2} & \cdot \sum_{l=0}^{2 q} \alpha_{l} \sin ^{2 l} x \cos ^{4 q-2 l} x \\
& \cdot \sum_{j=0}^{k-1}(-1)^{j}\left(\begin{array}{c}
k-1 \\
j
\end{array}\right) \sin ^{4 q j} x \cos ^{4(k-j-1) q} x d x
\end{aligned}
$$

and the result follows.

From this representation it is possible to deduce an explicit expression for the character sum $T(k, 4 q)$.

COROLlaRY 3.3 .

$$
T(k, 4 q)=-2 \cdot \frac{1+4 k q}{1+2 k q} \sum_{j=0}^{n}(-1)^{j}\left(\begin{array}{l}
k \\
j
\end{array}\right) \frac{\left(\frac{1}{2}\right)_{2 j q}}{\left(-\frac{1}{2}-2 k q\right)_{2 j q+1}}(k-2 j) q
$$

where $k=2 n+1$.

PrOOF. Let $J$ denote $\frac{1}{2} j p$, so $J \equiv 0 \bmod 2$. From Theorem 3.2

$$
\begin{aligned}
T(k, p) & =\frac{2}{\pi} \Delta \sum_{j=0}^{k}(-1)^{j}\left(\begin{array}{l}
k \\
j
\end{array}\right) \int_{0}^{\pi / 2}\left(\sin ^{2 J} x \cos ^{2(K-J+1)} x\right. \\
& \left.-\sin ^{2(J+1)} x \cos ^{2(K-J)} x\right) d x \\
& =\frac{\Delta}{4^{K+1}(K+1) !} \sum_{j=0}^{k}(-1)^{j}\left(\begin{array}{l}
k \\
j
\end{array}\right)\left(\Phi_{K+1}(J)-\Phi_{K+1}(J+1)\right),
\end{aligned}
$$

from Proposition 3.1. Now express $\Phi_{K+1}(J)$ and $\Phi_{K+1}(J+1)$ in terms of Pochhammer symbols in canonical form for the sum over $j$. This gives

$$
\begin{aligned}
T(k, p) & =\frac{1}{2(1+K)} \cdot \sum_{j=0}^{k}(-1)^{j}\left(\begin{array}{l}
k \\
j
\end{array}\right) \frac{\left(\frac{1}{2}\right)_{J}}{\left(-\frac{1}{2}-K\right)_{J}}\left\{\frac{\left(\frac{3}{2}\right)_{K}}{\left(\frac{1}{2}\right)_{K}}-\frac{\left(\frac{3}{2}\right)_{J}}{\left(\frac{1}{2}\right)_{J}} \cdot \frac{\left(-\frac{1}{2}-K\right)_{J}}{\left(\frac{1}{2}-K\right)_{J}}\right\} \\
& =\frac{1+2 K}{2(1+K)}\left\{\sum_{j=0}^{k}(-1)^{j}\left(\begin{array}{l}
k \\
j
\end{array}\right) \frac{\left(\frac{1}{2}\right)_{J}}{\left(-\frac{1}{2}-K\right)_{J}}-\sum_{j=0}^{k}(-1)^{j}\left(\begin{array}{l}
k \\
j
\end{array}\right) u(j, k)\right\}
\end{aligned}
$$

where

$$
u(j, k)=\frac{1+2 J}{1+2(K-J)} \cdot \frac{\left(\frac{1}{2}\right)_{J}}{\left(-\frac{1}{2}-K\right)_{J}} .
$$

It is a straightforward matter to show that $u(k-j, k)=\left(\frac{1}{2}\right)_{J} /\left(-\frac{1}{2}-K\right)_{J}$ whence

$$
T(k, p)=\frac{1+2 K}{2(1+K)}\left\{1-(-1)^{k}\right\} \sum_{j=0}^{k}(-1)^{j}\left(\begin{array}{l}
k \\
j
\end{array}\right) \frac{\left(\frac{1}{2}\right)_{J}}{\left(-\frac{1}{2}-K\right)_{J}} .
$$

Since $k \equiv 1 \bmod 2$

$$
T(k, 4 q)=\frac{1+4 k q}{1+2 k q} \sum_{j=0}^{k}(-1)^{j}\left(\begin{array}{l}
k \\
j
\end{array}\right) \frac{\left(\frac{1}{2}\right)_{2 j q}}{\left(-\frac{1}{2}-2 k q\right)_{2 j q}} .
$$


The result now follows by again using the fact that

$$
u(k-j, k)=\frac{\left(\frac{1}{2}\right)_{J}}{\left(-\frac{1}{2}-K\right)_{J}} .
$$

4. An integral representation for $T(4 n+2,2 q+1)$. An integral representation for the character sum $T(4 n+2,2 q+1)$ can be obtained in a similar way. The main points are contained in the next two propositions whose proofs are straightforward and are therefore omitted. Throughout this section, let $p=2 q+1$ and $k=4 n+2$.

PROPOSITION 4.1. Let

$$
v_{i}=(-1)^{i}\left(\begin{array}{c}
k p-1 \\
i
\end{array}\right)^{-1}\left\{\left[x^{i}\right](1+x)^{-1}\left(1-x^{2}\right)^{p k / 2}\right\} \cdot\left[x^{i}\right](1+x)^{-1}\left(1+x^{p}\right)^{k},
$$

and let $\rho^{\prime}$ be the map defined by

$$
\rho^{\prime}: \mathbf{N}_{2 n} \times \mathbf{N}_{q-1} \times \mathbf{N}_{1} \rightarrow \mathbf{N}_{k p-1}:(j, r, l) \mapsto 2(2 q+1) j+(2 q) l+2 r+1 .
$$

Then

(1) $\rho^{\prime}$ is injective.

(2) If $i=2(2 q+1) j+2 t+1$ where $0 \leq j \leq 2 n$ and $0 \leq t \leq 2 q-1$ then $v_{i+1}=v_{i}$.

(3) Let $i=\rho^{\prime}(j, r, l)$. Then

$v_{i}=\frac{K !}{(2 K) !} \cdot\left(\begin{array}{c}4 n+1 \\ 2 j+l\end{array}\right)(-1)^{1+j+l(q+1)+r} \Phi_{(2 q+1)(2 n+1)}((2 q+1) j+r+1+l q)$.

The next proposition follows from Lemma 2.3 and Proposition 4.1.

PROPOSITION 4.2. Let $\mu_{i, p}=1$ if $i=0$ or $p$, and $\mu_{i, p}=2$ otherwise. Then

$$
\begin{aligned}
T(k, p)=\frac{K !}{(2 K) !} \sum_{j=0}^{2 n}(-1)^{j} \sum_{l=0}^{1}\left(\begin{array}{c}
4 n+1 \\
2 j+l
\end{array}\right) \sum_{r=0}^{q}(-1)^{r+l q} \mu_{(q+1) l+r, p} \\
\cdot \Phi_{(2 n+1) p}(p j+r+(q+1) l) .
\end{aligned}
$$

This gives the following integral representation for the character sum. Here, and in Corollaries 5.2 and 6.2, $i$ denotes the complex number $\sqrt{-1}$ and $\mathbf{R}_{c}$ denotes the real part of $c \in \mathbb{C}$.

THEOREM 4.3.

$$
\begin{aligned}
& T(4 n+2,2 q+1) \\
& =\frac{4}{\pi} \Delta \cdot \mathbf{R} \int_{0}^{\pi / 2}\left\{2 \cos x\left(\cos ^{2(q+1)} x+(-1)^{q} \sin ^{2(q+1)} x\right)-\cos ^{2 q+1} x\right\} \\
& \cdot\left(\cos ^{2 q+1} x+i \sin ^{2 q+1} x\right)^{4 n+1} d x \text {. }
\end{aligned}
$$

PrOOF. From Propositions 3.1 and 4.2

$$
\begin{aligned}
T(4 n+2,2 q+1)=\frac{2}{\pi} & \Delta \sum_{l=0}^{1} \int_{0}^{\pi / 2} \sum_{r=0}^{q}(-1)^{r+l q} \mu_{(q+1) l+r, p} \sin ^{2 r+l} x \cos ^{p-2 r-l} x \\
& \cdot \sum_{j=0}^{2 n}(-1)^{j}\left(\begin{array}{c}
4 n+1 \\
2 j+l
\end{array}\right)\left(\sin ^{p} x\right)^{2 j+l}\left(\cos ^{p} x\right)^{4 n+1-(2 j+l)} d x .
\end{aligned}
$$


But the integral has the same value at $l=0,1$ so putting $l=0$ and simplifying the sum over $j$ we have

$$
\begin{array}{r}
T(4 n+2,2 q+1)=\frac{4}{\pi} \Delta \cdot \mathbf{R} \int_{0}^{\pi / 2} \sum_{r=0}^{q}(-1)^{r} \mu_{r, p} \sin ^{2 r} x \cos ^{2 q+1-2 r} x \\
\cdot\left(\cos ^{2 q+1} x+i \sin ^{2 q+1} x\right)^{4 n+1} d x .
\end{array}
$$

The trigonometrical sum is easily simplified to give the result.

An explicit expression for $T(4 n+2,2 q+1)$ can now be given.

COROLlaRY 4.4 .

$$
\begin{aligned}
T(k, p)=\frac{2}{K+1}\left\{\alpha(k, p)+\sum_{j=1}^{n}(K-\right. & 2 j p)\left(\begin{array}{c}
k \\
2 j
\end{array}\right) \frac{\left(\frac{1}{2}\right)_{j p}}{\left(\frac{1}{2}-K\right)_{j p}} \\
& \left.+\beta(k, p) \sum_{j=0}^{n-1}\left(\begin{array}{c}
k \\
2 j+1
\end{array}\right) \frac{\left(1+\frac{1}{2} p\right)_{j p}}{\left(-K+\frac{1}{2} p\right)_{j p}}\right\}
\end{aligned}
$$

where

$$
\beta(k, p)=2 \cdot\left(\frac{1}{2}\right)_{q+1} /\left(\frac{1}{2}-K\right)_{q}
$$

and

$$
\alpha(k, p)=K+\beta(k, p)\left(\begin{array}{c}
k-1 \\
\frac{1}{2} k
\end{array}\right) \frac{\left(1+\frac{1}{2} p\right)_{n p}}{\left(-K+\frac{1}{2} p\right)_{n p}} .
$$

ProOF. Let $J$ denote $\frac{1}{2} j p$. From Theorem $4.3, T(4 n+2,2 q+1)=4 \Delta I / \pi$ where

$$
\begin{array}{r}
I=\mathbf{R} \int_{0}^{\pi / 2}\left(2 \cos ^{2 q+3} x+2(-1)^{q} \cos x \sin ^{2 q+2} x-\cos ^{2 q+1} x\right) \\
\cdot\left(\cos ^{2 q+1} x+i \sin ^{2 q+1} x\right)^{4 n+1} d x .
\end{array}
$$

Next, we expand this, integrate it using Proposition 3.1 and reduce the Pochhammer symbols to canonical form, to obtain $I=\frac{1}{2} \pi I_{1}+\frac{1}{2} \pi \beta(k, q) I_{2}$ where

$$
I_{1}=\frac{\left(\frac{1}{2}\right)_{K}}{(K+1) !} \sum_{j=0}^{2 n}\left(\begin{array}{c}
4 n+1 \\
2 j
\end{array}\right) \frac{\left(\frac{1}{2}\right)_{2 J}}{\left(\frac{1}{2}-K\right)_{2 J}}(K-4 J)
$$

and

$$
I_{2}=2 \cdot \sum_{j=0}^{2 n}\left(\begin{array}{c}
4 n+1 \\
2 j
\end{array}\right) \frac{\left(\frac{1}{2} p+1\right)_{2 J}}{\left(\frac{1}{2} p-K\right)_{2 J}} .
$$

For $I_{1}$, let $j=2 n+1-j^{\prime}$ and let $J^{\prime}$ denote $\frac{1}{2} p j^{\prime}$ so $2 J=K-2 J^{\prime}$. It is a routine matter to check that

$$
\left(\begin{array}{c}
4 n+1 \\
2 j^{\prime}
\end{array}\right)=\left(\begin{array}{c}
4 n+1 \\
2 j-1
\end{array}\right) \text { and } \frac{\left(\frac{1}{2}\right)_{2 J^{\prime}}}{\left(\frac{1}{2}-K\right)_{2 J^{\prime}}}=-\frac{\left(\frac{1}{2}\right)_{2 J}}{\left(\frac{1}{2}-K\right)_{2 J}}
$$

whence

$$
I_{1}=K+\sum_{j=0}^{n}(K-4 J)\left(\begin{array}{c}
4 n+2 \\
2 j
\end{array}\right) \frac{\left(\frac{1}{2}\right)_{2 J}}{\left(\frac{1}{2}-K\right)_{2 J}}
$$


For $I_{2}$, let $j=2 n-j^{\prime \prime}$ and let $J^{\prime \prime}$ denote $\frac{1}{2} p j^{\prime \prime}$ so $2 J=K-p-2 J^{\prime \prime}$. Again, it is readily seen that

$$
\left(\begin{array}{c}
4 n+1 \\
2 j^{\prime \prime}
\end{array}\right)=\left(\begin{array}{c}
4 n+1 \\
2 j+1
\end{array}\right) \text { and } \frac{\left(1+\frac{1}{2} p\right)_{2 J^{\prime \prime}}}{\left(\frac{1}{2} p-K\right)_{2 J^{\prime \prime}}}=\frac{\left(1+\frac{1}{2} p\right)_{2 J}}{\left(\frac{1}{2} p-K\right)_{2 J}}
$$

whence

$$
I_{2}=2 \cdot\left\{\left(\begin{array}{c}
4 n+1 \\
2 n
\end{array}\right) \frac{\left(1+\frac{1}{2} p\right)_{n p}}{\left(-K+\frac{1}{2} p\right)_{n p}}+\sum_{j=0}^{n-1}\left(\begin{array}{c}
4 n+2 \\
2 j+1
\end{array}\right) \frac{\left(1+\frac{1}{2} p\right)_{2 J}}{\left(-K+\frac{1}{2} p\right)_{2 J}}\right\}
$$

which gives the result.

5. Some particular values of the character sum. The integral representation of $T(k, p)$ may be used to obtain explicit expressions in certain special cases.

COROLLARY 5.1 .

$$
T(2 n-1,4)=4^{3 n-2}\left(\begin{array}{c}
2 n \\
n
\end{array}\right)\left(\begin{array}{c}
8 n-4 \\
4 n-2
\end{array}\right)^{-1} .
$$

PrOOF. From Theorem 3.2

$$
\begin{aligned}
T(2 n-1,4) & =4^{2(2 n-1)} \cdot \frac{2}{\pi}\left(\begin{array}{c}
8 n-4 \\
4 n-2
\end{array}\right)^{-1} \int_{0}^{\pi / 2}\left(\cos ^{4} x-\sin ^{4} x\right)^{2 n-1} \cos 2 x d x \\
& =4^{2(2 n-1)}\left(\begin{array}{c}
8 n-4 \\
4 n-2
\end{array}\right)^{-1} \frac{2}{\pi} \int_{0}^{\pi / 2} \cos ^{2 n} 2 x d x .
\end{aligned}
$$

The result follows from Proposition 3.1.

This agrees with [2].

COROLLARY 5.2.

$$
T(4 n+2,3)=12\left(2^{4} \cdot 3^{3}\right)^{n}\left(\begin{array}{c}
4 n+2 \\
n+1
\end{array}\right)\left(\begin{array}{c}
12 n+6 \\
6 n+3
\end{array}\right)^{-1} .
$$

PrOOF. From Theorem 4.3

$$
\begin{aligned}
& T(4 n+2,3)=\left(\begin{array}{c}
12 n+6 \\
6 n+3
\end{array}\right)^{-1} 4^{6 n+3} \\
& \cdot \frac{1}{\pi} \mathbf{R} \int_{0}^{\pi / 2}(3 \cos 3 x+\cos x)\left(\cos ^{3} x+i \sin ^{3} x\right)^{4 n+1} d x .
\end{aligned}
$$

Now

$$
\begin{aligned}
\mathbf{R} \int_{0}^{\pi / 2} & (3 \cos 3 x+\cos x)\left(\cos ^{3} x+i \sin ^{3} x\right)^{4 n+1} d x \\
= & \frac{1}{2} \mathbf{R} \frac{1}{4^{4 n+1}} \int_{0}^{\pi / 2}\left(3 e^{3 i x}+3 e^{-3 i x}+e^{i x}+e^{-i x}\right) e^{(4 n+1) i x}\left(3+e^{-4 i x}\right)^{4 n+1} d x \\
= & \frac{1}{2 \cdot 4^{4 n+1}} \cdot \sum_{j=0}^{4 n+1}\left(\begin{array}{c}
4 n+1 \\
j
\end{array}\right) 3^{4 n+1-j}\left(3 \lambda_{j}(4)+3 \lambda_{j}(-2)+\lambda_{j}(2)+\lambda_{j}(0)\right)
\end{aligned}
$$

where

$$
\lambda_{j}(\theta)=\mathbf{R} \int_{0}^{\pi / 2} e^{(4(n-j)+\theta) i x} d x=\frac{1}{2} \pi \delta_{\theta / 4+n, j},
$$


provided $\theta$ is even. But $\lambda_{j}(-2)=\lambda_{j}(2)=0$ so

$$
\begin{aligned}
\mathbf{R} \int_{0}^{\pi / 2} & (3 \cos 3 x+\cos x)\left(\cos ^{3} x+i \sin ^{3} x\right)^{4 n+1} d x \\
= & \frac{1}{2 \cdot 4^{4 n+1}} \sum_{j=0}^{4 n+1}\left(\begin{array}{c}
4 n+1 \\
j
\end{array}\right) 3^{4 n+1-j} \cdot \frac{\pi}{2}\left(3 \cdot \delta_{1+n, j}+\delta_{n, j}\right) \\
= & \frac{3^{3 n+1}}{4^{4 n+2}}\left(\begin{array}{c}
4 n+2 \\
n+1
\end{array}\right) \pi
\end{aligned}
$$

and the result follows.

6. Recurrence equations. We next show how an explicit recurrence equation can be obtained for $T(2 n-1,8)$, by using the contiguous relations of Gauss for the ${ }_{2} F_{1}$ (Rainville [7]).

COROLlary 6.1. Let

$$
T(2 n-1,8)=2 \cdot 4^{6 n-4}\left(\begin{array}{c}
2 n \\
n
\end{array}\right)\left(\begin{array}{c}
16 n-8 \\
8 n-4
\end{array}\right)^{-1} b_{n}
$$

Then $\left\{b_{n} \mid n \geq 1\right\}$ satisfies the 3 -term linear recurrence relation

$$
\begin{aligned}
12(n+1) & (3 n+4)(3 n+5)(8 n+1) b_{n+2} \\
= & (n+2)\left(3328 n^{3}+7072 n^{2}+4104 n+435\right) b_{n+1} \\
& +64 n(n+1)(n+2)(8 n+9) b_{n}
\end{aligned}
$$

with the initial conditions $b_{1}=7 / 4, b_{2}=203 / 32$.

Proof. From Theorem 3.2

$$
\begin{aligned}
T(2 n-1,8) & =4^{4(2 n-1)}\left(\begin{array}{c}
16 n-8 \\
8 n-4
\end{array}\right)^{-1} \frac{2}{\pi} \int_{0}^{\pi / 2}\left(\cos ^{8} x-\sin ^{8} x\right)^{2 n-1} \cos 2 x d x \\
& =4^{7 n-4}\left(\begin{array}{c}
16 n-8 \\
8 n-4
\end{array}\right)^{-1} \frac{2}{\pi} \int_{0}^{\pi}\left(1+\cos ^{2} x\right)^{2 n-1} \cos ^{2 n} x d x .
\end{aligned}
$$

Expanding this and using Proposition 3.1 gives

$$
\begin{aligned}
T(2 n-1,8) & =2 \cdot 4^{6 n-4}\left(\begin{array}{c}
16 n-8 \\
8 n-4
\end{array}\right) \sum_{j=0}^{-1}\left(\begin{array}{c}
2 n-1 \\
j
\end{array}\right)\left(\begin{array}{c}
2(j+n) \\
j+n
\end{array}\right) / 4^{j} \\
& =2 \cdot 4^{6 n-4}\left(\begin{array}{c}
2 n \\
n
\end{array}\right)\left(\begin{array}{c}
16 n-8 \\
8 n-4
\end{array}\right)^{-1} b_{n}
\end{aligned}
$$

where

$$
b_{n}={ }_{2} F_{1}\left[\begin{array}{c}
1-2 n, \frac{1}{2}+n \\
-, 1+n
\end{array}\right],
$$

thereby identifying $b_{n}$ as Gauss' function. Let

$$
F={ }_{2} F_{1}\left[\begin{array}{c}
a, b \\
-, c
\end{array} \mid x\right] \text { and } F[a \pm 1]={ }_{2} F_{1}\left[\begin{array}{c}
a \pm 1, b \\
-, c
\end{array} \mid x\right]
$$


and similarly for $F[b \pm 1], F[c \pm 1]$. Among the relationships satisfied by these contiguous series are the following (Slater [9])

$$
\begin{gathered}
(c-a) F[a-1]=\{c-2 a+(a-b) x\} F+a(1-x) F[a+1], \\
(c-a)(c-b) x F[c+1]=a c(1-x) F[a+1]-c\{a+(b-c) x\} F, \\
(c-a) F[a-1]=(b-a)(1-x) F+(c-b) F[b-1], \\
b F[b+1]=-(c-b-1) F+(c-1) F[c-1] .
\end{gathered}
$$

Let

$$
\begin{gathered}
\left.A_{n}={ }_{2} F_{1}\left[\begin{array}{c}
-1-2 n, \frac{1}{2}+n \\
-, 2+n
\end{array} \mid-1\right], \quad B_{n}={ }_{2} F_{1}\left[\begin{array}{c}
-1-2 n, \frac{1}{2}+n \\
-, 1+n
\end{array}\right]-1\right] \\
Z_{n}={ }_{2} F_{1}\left[\begin{array}{c}
-2 n, \frac{1}{2}+n \\
-, 1+n
\end{array} \mid-1\right] .
\end{gathered}
$$

Then these satisfy the following

$$
\begin{gathered}
-A_{n}+2(1+n) B_{n}=(1+2 n) b_{n+1} \quad \text { from }(4) \\
(2+3 n) A_{n}+(1+n)(1+4 n) B_{n}-4(1+n)(1+2 n) Z_{n}=0 \quad \text { from }(2) \\
2(1+3 n) B_{n}-(16 n+3) Z_{n}=-8 n b_{n} \quad \text { from }(1) \\
A_{n}-6(1+n) Z_{n+1}=-2(5+6 n) b_{n+1} \quad \text { from }(3)
\end{gathered}
$$

Elimination of $B_{n}, Z_{n}$ between (5), (6), (7) gives

$$
\left(16 n^{2}-12 n-5\right) b_{n+1}-64 n(n+1) b_{n}+7(8 n+1) A_{n}=0 \text {. }
$$

Elmination of $A_{n}, B_{n}$ between (5), (6), (7) gives

$$
7(n+1)(8 n+1) Z_{n}-2(3 n+1)(3 n+2) b_{n+1}-40 n(n+1) b_{n}=0
$$

which gives, on replacement of $n$ by $n+1$,

(10) $7(n+2)(8 n+9) Z_{n+1}-2(3 n+4)(3 n+5) b_{n+2}-40(n+1)(n+2) b_{n+1}=0$.

The recurrence equation follows by eliminating $A_{n}$ and $Z_{n+1}$ between (8), (9), (10). The initial conditions are obtained by direct calculation of $b_{1}$ and $b_{2}$.

In fact, it is readily shown that the initial conditions $b_{0}=0, b_{1}=7 / 4$ generate the same sequence. The selection of the value of $b_{0}$ is arbitrary. cult.

The task of obtaining a linear recurrence equation for $T(4 n+2,5)$ is more diffi-

COROLLARY 6.2. Let

$$
T(4 n+2,5)=\frac{2^{5} \cdot 5^{2}(2 n+1)\left(2^{7} \cdot 5^{4}\right)^{n}\left(\begin{array}{c}
4 n+1 \\
n
\end{array}\right)}{(3 n+2)\left(\begin{array}{c}
20 n+10 \\
10 n+5
\end{array}\right)} c_{n}
$$


Then $\left\{c_{n} \mid n \geq 0\right\}$ satisfies the 3-term linear recurrence relation

$$
\begin{gathered}
5^{4}(5 n-2)(5 n-1)(5 n+1)(5 n+2)\left(2800 n^{3}-7000 n^{2}+5692 n-1503\right) c_{n} \\
=2 \cdot 5^{3}\left(5824000 n^{7}-14560000 n^{6}+10831360 n^{5}-652000 n^{4}\right. \\
\left.\quad-1952864 n^{3}+465176 n^{2}+32736 n-7623\right) c_{n-1} \\
\quad+2^{10} \cdot 3(n-1)^{2}(3 n-2)(3 n-1)\left(2800 n^{3}+1400 n^{2}+92 n-11\right) c_{n-2}
\end{gathered}
$$

with the initial conditions $c_{0}=11 / 10, c_{1}=61 / 40$.

PROOF. From Theorem 4.3

$$
T(4 n+2,5)=\left(\begin{array}{c}
20 n+10 \\
10 n+5
\end{array}\right)^{-1} 4^{5(2 n+1)} \frac{4}{\pi} I^{\prime}
$$

where

$$
\begin{aligned}
& I^{\prime}=\mathbf{R} \int_{0}^{\pi / 2}\left(2 \cos ^{7} x+2 \cos x \sin ^{6} x-\cos ^{5} x\right)\left(\cos ^{5} x+i \sin ^{5} x\right)^{4 n+1} d x \\
& =\frac{1}{2\left(4^{2}\right)^{4 n+2}} \mathbf{R} \int_{0}^{\pi / 2}\left(5 e^{5 i x}+e^{3 i x}+10 e^{i x}+10 e^{-i x}+e^{-3 i x}+5 e^{-5 i x}\right) \\
& \cdot e^{(4 n+1) i x}\left(e^{4 i x}+5 e^{-4 i x}+10\right)^{4 n+1} d x \\
& =\frac{1}{2\left(4^{2}\right)^{4 n+2}} \mathbf{R} \int_{0}^{\pi / 2} \sum_{\substack{r, s, t \geq 0 \\
r+s+t=4 n+1}} 5^{s} 10^{t}\left[\begin{array}{c}
4 n+1 \\
r, s, t
\end{array}\right] \\
& \sum_{\lambda \in\{6,4,2,0,-2,-4\}} a_{\lambda} e^{(4(n+r-s)+\lambda) i x} d x \\
& \text { where } a_{-4}=5, a_{-2}=1, a_{0}=10, a_{2}=10, a_{4}=1, a_{6}=5
\end{aligned}
$$

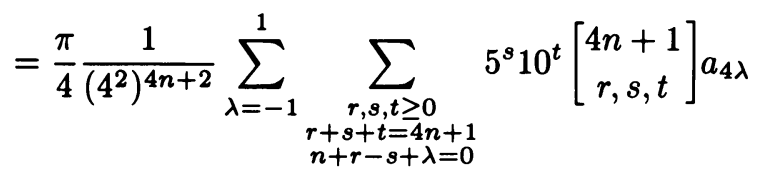

since

$$
\mathbf{R} \int_{0}^{\pi / 2} e^{(4(n+r-s)+\lambda) i x} d x=\frac{\pi}{2} \delta_{4(n+r-s)+\lambda, 0}
$$

provided $\lambda$ is even. In the preceding expressions

$$
\left[\begin{array}{c}
j \\
j_{1}, j_{2}, j_{3}
\end{array}\right] \text { denotes } j ! /\left(j_{1} ! j_{2} ! j_{3} !\right)
$$

Thus

$$
\begin{gathered}
I^{\prime}=\frac{\pi}{4} \frac{1}{\left(4^{2}\right)^{4 n+2}} \sum_{\lambda=-1}^{1} \sum_{r \geq 0} 5^{r+n+\lambda} \cdot 10^{3 n-2 r+1-\lambda}\left[\begin{array}{c}
4 n+1 \\
r, r+n+\lambda, 3 n-2 r+1-\lambda
\end{array}\right] a_{4 \lambda} \\
=\frac{\pi}{4} \frac{10}{\left(4^{2}\right)^{2}}\left(\frac{5 \cdot 10^{3}}{4^{8}}\right)^{n} \sum_{\lambda=-1}^{1} \frac{1}{2^{\lambda}} a_{4 \lambda}\left(\begin{array}{c}
4 n+1 \\
n+\lambda
\end{array}\right) \\
\cdot{ }_{2} F_{1}\left[\begin{array}{c}
-\frac{1}{2}-3 n / 2+\lambda / 2,-3 n / 2+\lambda / 2 \\
-, 1+n+\lambda
\end{array}\right],
\end{gathered}
$$


where, for these values of $\lambda$, the ${ }_{2} F_{1}$ 's are naturally terminating.

Let

$$
\begin{gathered}
G_{n}={ }_{2} F_{1}\left[\begin{array}{c}
-3 n / 2,-1 / 2-3 n / 2 \\
-, 1+n
\end{array} \mid \begin{array}{c}
1 / 5 \\
H_{n}
\end{array}={ }_{2} F_{1}\left[\begin{array}{c}
-3 n / 2,1 / 2-3 n / 2 \\
-, 1+n
\end{array} \mid 1 / 5\right]\right.
\end{gathered}
$$

and

$$
c_{n}={ }_{2} F_{1}\left[\begin{array}{c}
-1-3 n / 2,-1 / 2-3 n / 2 \\
-, 1+n
\end{array} \mid 1 / 5\right] \text {. }
$$

We use the following contiguous relations:

$$
\begin{gathered}
(c-1) F[c-1]=(c-a-1) F+a F[a+1], \\
(c-a) x F[c+1]=c F[b-1]-c(1-x) F, \\
(c-a) F[a-1]=(c-a-b) F+b(1-x) F[b+1]
\end{gathered}
$$

in the notation given in the proof of Corollary 6.1.

From (1), (2) and (3) respectively

$$
\begin{aligned}
& 2 n_{2} F_{1}\left[\begin{array}{c}
-1-3 n / 2,-1 / 2-3 n / 2 \\
-, n
\end{array}\right]=(5 n+2) c_{n}-(3 n+2) G_{n}, \\
& (5 n+2)_{2} F_{1}\left[\begin{array}{c}
-3 n / 2,1 / 2-3 n / 2 \\
-, 2+n
\end{array} \mid 1 / 5\right]=10(n+1) G_{n}-8(n+1) H_{n}, \\
& 5(5 n+2) c_{n}=5(8 n+3) G_{n}-4(3 n+1) H_{n} .
\end{aligned}
$$

From (4) and (5)

$$
\begin{aligned}
T(4 n+2,5)= & 2^{3} \cdot 5\left(\begin{array}{c}
20 n+10 \\
10 n+5
\end{array}\right)^{-1}\left(2^{7} \cdot 5^{4}\right)^{n} \\
& \cdot\left\{\frac{5}{n}\left(\begin{array}{c}
4 n+1 \\
n-1
\end{array}\right)\left((5 n+2) c_{n}-(3 n+2) G_{n}\right)+10\left(\begin{array}{c}
4 n+1 \\
n
\end{array}\right) G_{n}\right. \\
= & \left.2^{3} \cdot 5 \cdot\left(\begin{array}{c}
4 n+1 \\
n+1
\end{array}\right)\left(5(n+1) G_{n}-4(n+1) H_{n}\right)\right\} \\
& \cdot\left\{\frac{5(5 n+2)}{3 n+2} c_{n}+\frac{1}{5 n+2}\left(5(8 n+3) G_{n}-4(3 n+1) H_{n}\right)\right\} \\
= & \frac{2^{5} \cdot 5^{2}(2 n+1)\left(2^{7} \cdot 5^{4}\right)^{n}\left(\begin{array}{c}
4 n+1 \\
n
\end{array}\right)}{(3 n+2)\left(\begin{array}{c}
20 n+10 \\
10 n+5
\end{array}\right)} \quad \text { from }(6) .
\end{aligned}
$$

We can now obtain a linear recurrence relation for $c_{n}$ by using the following contiguous relations:

$$
(c-a)(c-b) x F[c+1]=b c(1-x) F[b+1]-c\{b+(a-c) x\} F,
$$




$$
\begin{gathered}
(a-1-(c-b-1) x) F+(c-a) F[a-1]-(c-1)(1-x) F[c-1]=0, \\
(c-a) F[a-1]=\{c-2 a+(a-b) x\} F+a(1-x) F[a+1] .
\end{gathered}
$$

Let $P_{n}={ }_{2} F_{1}\left[\begin{array}{c}1-3 n / 2,-1 / 2-3 n / 2 \\ -, n\end{array} \mid 1 / 5\right], Q_{n}={ }_{2} F_{1}\left[\begin{array}{c}1-3 n / 2,-1 / 2-3 n / 2 \\ -, 1+n\end{array} \mid 1 / 5\right]$. Then

$$
\begin{gathered}
(5 n-2)(5 n+1) Q_{n}=-8(3 n+1) n c_{n-1}+2 n(20 n+3) P_{n} \text { from (7), } \\
-(20 n+1) Q_{n}+25 n G_{n}-8 n P_{n}=0 \text { from (8), } \\
5(5 n+2) c_{n}=(40 n+11) G_{n}-12 n Q_{n} \quad \text { from (9), } \\
5(5 n-1) P_{n}=(40 n-11) c_{n-1}-4(3 n-1) G_{n-1} \quad \text { from (9). }
\end{gathered}
$$

Elimination of $Q_{n}, G_{n}$ between (10), (11), (12) gives

$$
\begin{aligned}
5^{3}(5 n-2)(5 n+1)(5 n+2) c_{n}= & 10\left(2800 n^{3}+1400 n^{2}+92 n-11\right) P_{n} \\
& -8(3 n+1)\left(500 n^{2}+260 n+11\right) c_{n-1} .
\end{aligned}
$$

Elimination of $P_{n}, Q_{n}$ between (10), (11), (12) gives

$$
\begin{aligned}
2^{7} \cdot 3 n^{2}(3 n+1) c_{n-1}= & 5^{2}(5 n+2)\left(100 n^{2}+12 n-1\right) c_{n} \\
& -5\left(2800 n^{3}+1400 n^{2}+92 n-11\right) G_{n},
\end{aligned}
$$

which gives, on replacement of $n$ by $n-1$,

$$
\begin{aligned}
2^{7} \cdot 3(n-1)^{2}(3 n-2) c_{n-2}= & 5^{2}(5 n-3)\left(100 n^{2}-188 n+87\right) c_{n-1} \\
& -5\left(2800 n^{3}-7000 n^{2}+5692 n-1503\right) G_{n-1} .
\end{aligned}
$$

The recurrence equation follows by eliminating $P_{n}$ and $G_{n-1}$ between (13), (14), (15). The initial conditions are obtained by direct calculation of $c_{0}, c_{1}$.

7. Concluding comments. The question of counting $p$-semiregular permutations in $\mathbf{S}_{n}$ which are expressible as the product of a full cycle and a $t$-semiregular permutation is also of combinatorial interest. However, for cases other than $t=1$, which is trivial, $t=2$, which has been treated here, and $t=n$ this problem seems to be intractable.

The expression for $T(4 n+2,3)$ given in Corollary 5.2 was conjectured from evidence drawn from computer computations based on Lemma 2.3. The eliminations needed to derive the recurrence equations for $T(2 n-1,8)$ and $T(4 n+2,5)$ given in Corollaries 6.1 and 6.2, were also carried out by means of a computer. In both instances, the computations were done symbolically using the Sun Unix Macsyma Beta Test Release 308.2 in the Department of Mathematics at MIT.

ACKNOWLEDGMENTS. This work was carried out during visits by the author to the Mathematics Department at MIT during May and July 1986, and was supported by a grant (A8235) from the National Sciences and Engineering Research Council of Canada.

Appendix. Figure 1a gives the map corresponding to the vertex permutation

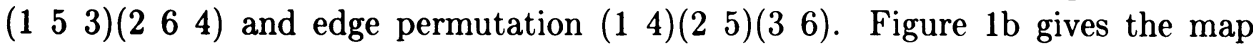
corresponding to the vertex permutation $\left(\begin{array}{lllll}1 & 8 & 11 & 6\end{array}\right)\left(\begin{array}{lllll}2 & 5 & 12 & 7\end{array}\right)\left(\begin{array}{llll}3 & 10 & 9 & 4\end{array}\right)$ and the edge permutation $\left(\begin{array}{lll}1 & 7\end{array}\right)\left(\begin{array}{lll}2 & 4\end{array}\right)\left(\begin{array}{ll}3 & 9\end{array}\right)\left(\begin{array}{lll}5 & 11\end{array}\right)\left(\begin{array}{ll}6 & 12\end{array}\right)\left(\begin{array}{ll}8 & 10\end{array}\right)$. Figures $2 \mathrm{a}$ and $2 \mathrm{~b}$ give a representation of the single face $\left(\begin{array}{llll}1 & 2 & \cdots & 6\end{array}\right)$ and $\left(\begin{array}{llll}1 & 2 & \cdots & 12\end{array}\right)$, respectively, for these maps by cutting along each edge. 


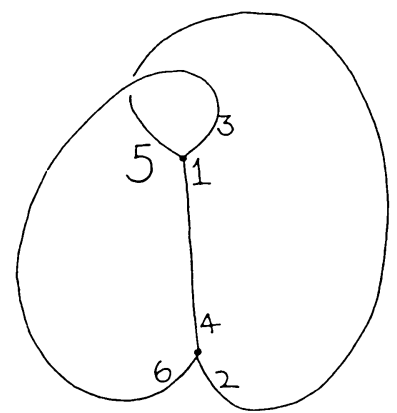

(a)

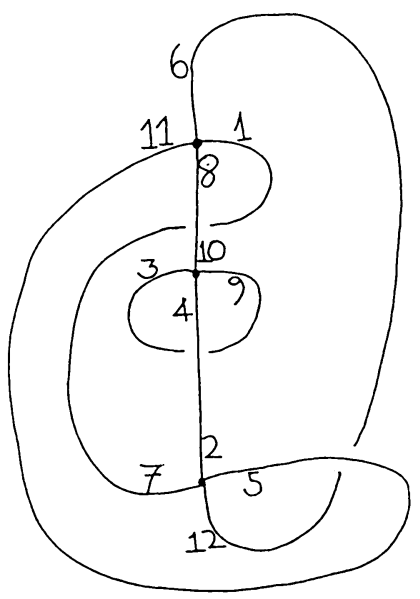

(b)

FIgURE 1. The map given in Figure 1a has genus one, while the map in Figure 2a has genus two.

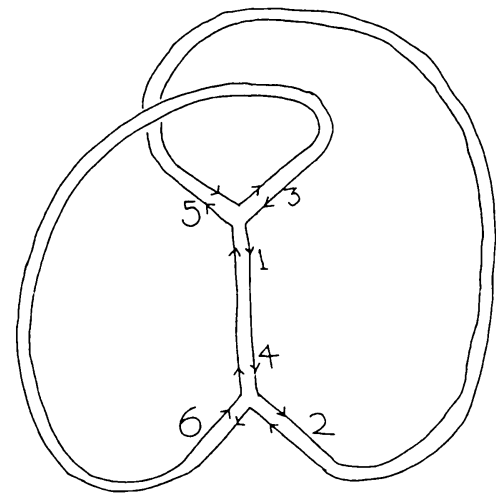

(a)

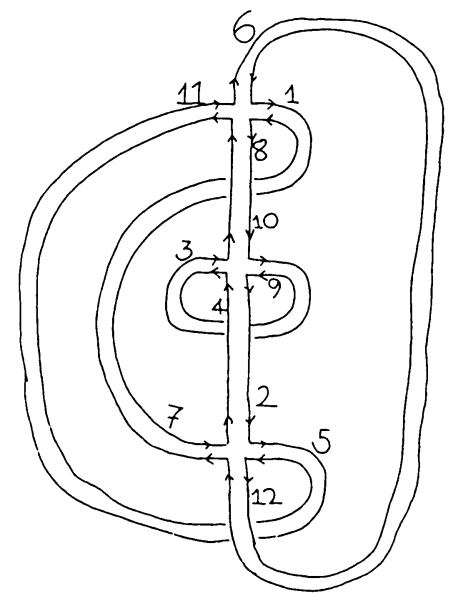

(b)

FIGURE 2. The face of the map given in Figure 1a is redrawn in Figure 2a, and is encoded by the permutation $(12 \cdots 6) \in \mathbf{S}_{6}$. The face of the map given in Figure $1 \mathrm{~b}$ is redrawn in Figure $2 \mathrm{~b}$, and is encoded by the permutation $(12 \cdots 12) \in \mathbf{S}_{12}$. Each permutation gives a directed cycle corresponding to a face boundary.

\section{REFERENCES}

1. E. Bannai and T. Ito, Algebraic combinatorics. I: Association schemes, Benjamin/Cummings, Menlo Park, California, 1984.

2. D. Bessis, C. Itzykson and J. B. Zuber, Quantum field theory techniques in graphical enumeration, Adv. Appl. Math. 1 (1980), 109-157. 
3. P. Diaconis and M. Shahshahani, Generating a random permutation with random transpositions, Z. Wahrsch. Geb. 57 (1981), 159-179.

4. D. M. Jackson, Counting cycles in permutations by group characters, with an application to a topological problem, Trans. Amer. Math. Soc. 299 (1987), 785-801.

5. A. Machi, The Riemann-Hurwitz formula for the centralizer of a pair of permutations, Arch. Math. 42 (1984), 280-288.

6. Private communication.

7. E. D. Rainville, Special functions, Macmillan, New York, 1960.

8. O. Rothaus and J. G. Thompson, A combinatorial problem in the symmetric group, Pacific J. Math. 18 (1966), 175-178.

9. L. J. Slater, Generalized hypergeometric functions, Cambridge Univ. Press, Cambridge, 1966.

10. R. P. Stanley, Factorization of permutations into $n$-cycles, Discrete Math. 37 (1981), 255-262.

11. J. G. Thompson, Rational functions associated to presentations of finite groups, J. Algebra 71 (1981), 481-489.

DEPARTMENT OF COMBINATORICS AND OPTIMIZATION, UNIVERSITY OF WATERLOO, WATERloO, ONTARIO, CANADA N2L 3G1 